\title{
Gradhiva
}

GRADHIV

Revue d'anthropologie et d'histoire des arts

Présence africaine

\section{Donne le change et change la donne}

\section{Ralph Ellison}

Traducteur : Emmanuel Parent

\section{OpenEdition}

\section{Journals}

\section{Édition électronique}

URL : http://journals.openedition.org/gradhiva/1589

DOI : 10.4000/gradhiva.1589

ISSN : 1760-849X

\section{Éditeur}

Musée du quai Branly Jacques Chirac

\section{Édition imprimée}

Date de publication : 4 novembre 2009

Pagination : 193-203

ISBN : 978-2-35744-012-8

ISSN : 0764-8928

\section{Référence électronique}

Ralph Ellison, «Donne le change et change la donne », Gradhiva [En ligne], 10 | 2009, mis en ligne le 04 novembre 2012, consulté le 21 avril 2019. URL : http://journals.openedition.org/gradhiva/1589 ; DOI : 10.4000/gradhiva.1589 


\section{Donne le change et change la donne}

\section{Ralph Ellison}

-

1. Ce texte, dont le titre original est Change the Joke and Slip the Yoke, a paru dans The Partisan Review au printemps 1958. (N. d. É.)

2. Voir supra, p. 182-192.

3. II s'agit de Invisible Man (1952), paru en français sous le titre Homme invisible, pour qui chantestu ? (Grasset, 1969). (N. d. É.)
J'éprouve pour les questions touchant à la relation entre la littérature et la tradition folklorique noires américaines le plus vif intérêt ${ }^{1}$. Aussi, à peine avais-je eu vent de l'essai de Stanley Edgar Hyman sur le sujet ${ }^{2}$ que ma bienveillance lui était déjà presque acquise. Pourtant, au fil de la lecture, je me rendis compte du profond désaccord qui nous séparait. Comment la tradition vernaculaire s'immisce-t-elle dans la littérature, et dans le roman en particulier? Ou'est-ce qui est proprement nègre, et qu'est-ce qui est américain dans le folklore noir? Quel est l'environnement de l'écrivain noir? Toutes ces questions nous divisent profondément. En outre, son essai s'appuyant si généreusement sur mes humbles écrits, je me retrouve dans une position assez ingrate, et pour tout dire embarrassante. Non seulement je dois répondre à ses affirmations en puisant dans ce sanctuaire si discutable (bien que privilégié) que constituent la subjectivité et l'histoire personnelle, mais il me faut également remettre en cause quelquesunes de ses analyses sur mon propre roman ${ }^{3}$, en revenant au texte.

Les archétypes, comme les impôts, semblent devoir nous poursuivre sans cesse. La littérature n'échappe vraisemblablement pas à cette maxime. Mais, entre l'archétype et l'œuvre d'art, il y a pourtant l'être humain en chair et en os, pris dans une texture spécifique de temps, de lieu et de circonstances, qui doit réagir, faire des choix, maîtriser son style et créer des œuvres uniques. C'est pourquoi il me semble que la fascination de Hyman pour la tradition vernaculaire, et l'ivresse que lui procure la traque des archétypes, le conduisent finalement à ignorer la spécificité des œuvres littéraires. Il estompe ce faisant la distinction entre plusieurs archétypes et entre différents courants du folklore américain, et, d'une manière générale, il finit par simplifier à outrance la tradition américaine.

Le personnage archétypique dont Hyman fait le plus grand cas est celui du décepteur, celui qui embobine et joue des tours. Mais j'y vois un danger. Car, d'un certain point de vue, tous les archétypes peuvent être vus comme des décepteurs ou des escrocs : moitié dieu, moitié homme, personne ne semble savoir quelle est sa vraie nature, car il, elle ou cela, est inconstant et change sans cesse de lieu, d'allure ou d'identité. En outre, le décepteur est partout et nulle part à la fois et, comme les parties de quelque dieu démembré, il peut surgir tout aussi bien d'un endroit rocailleux que d'une plaine fertile. Le folklore est sans doute plus stable, dans son identité si ce n'est dans sa généalogie. Mais même là, si nous devons examiner le folklore noir américain, gardons notre sang-froid et ne permettons pas à quelques intrus de nous induire en erreur.

En premier lieu, il faudrait éviter d'aborder le folklore noir au travers de cette figure que Hyman nomme "l'amuseur noir " ["darky" entertainer]. Car, même si 
les artistes qu'il convoque apparaissent de prime abord comme des guides fort commodes, ils nous introduisent finalement dans un labyrinthe chtonien où nous nous perdons bientôt. En dépit de sa «noirceur», le personnage auquel ils renvoient n'est pas le Noir américain (et, de fait, il inspire aux Noirs un dégoût profond). Malgré sa ressemblance avec le clown cher au public noir des spectacles de variétés, cette figure, populaire auprès des Blancs, ne l'est pas parmi les Afro-Américains, car elle dérive en réalité de la branche anglo-saxonne du folklore américain. En d'autres termes, cet amuseur noir est blanc. Mais qu'importe; suivons sa piste un instant, et même si nous sommes amenés à traiter davantage de problèmes raciaux que de littérature, nous n'aurons vraisemblablement pas perdu notre temps.

Ces amuseurs sont, comme l'explique Hyman, des professionnels endossant un masque rituel pour jouer un rôle symbolique qui sous-tend toute la comédie sociale américaine - ce masque que les ménestrels blancs arboraient lorsqu'ils imitaient des comiques noirs. Depuis les années 1930, les changements sociaux ont quelque peu modifié la donne (Rochester, par exemple, évolue dans un climat rhétorique assez différent, disons, de celui d'un Stepin Fetchit4). Mais le masque, si emblématique et stylisé, devait toujours rester de mise pour quiconque voulait tenir ce rôle. Il s'imposait même pour les Noirs, qui, abstraction faite de la dimension rituelle, auraient pourtant dû en être dispensés du fait de leur taux mélanique naturellement élevé.

Notons également que le rôle qui met en scène le parler, les chansons, les danses et les jeux de mots noirs ne s'appuie pas sur un sens de l'humour typiquement noir (bien que nous ayons nous aussi notre comédie de la noirceur), mais plutôt sur la fascination des Blancs américains pour le symbolisme de la noirceur et de la blancheur. On en voit par exemple l'expression dans les contradictions entre la moralité judéo-chrétienne de l'Amérique blanche, ses idéaux politiques démocratiques et sa conduite au quotidien - en un mot, son rapport au monde délibérément anti-tragique.

Être associé au côté négatif du dualisme basique de la mentalité occidentale du fait de notre "forte pigmentation", comme disent les sociologues, et être enchaîné à tout ce que réprime la conscience blanche, voilà le terrible accident, notre noire «infortune ». Les souffrances physiques et les humiliations de l'esclavage étaient en vérité peu de chose en regard de cette dévalorisation perpétuelle de notre image. Il est pour de nombreux Blancs quasiment impossible d'envisager les questions du sexe, des femmes, de l'argent, de l'identité nationale, du changement historique, de la justice sociale, et même la "criminalité » accompagnant nécessairement l'accroissement des libertés - toutes ces choses ayant partie liée avec leur représentation du chaos -, sans automatiquement y associer des images malignes et inquiétantes de Nègres.

Dans la branche anglo-saxonne du folklore américain et dans l'industrie du divertissement (qui se nourrit de l'exploitation et de la dépréciation de tous les matériaux folkloriques), le Noir est réduit à un signe négatif qui apparaît généralement dans une comédie mettant en scène le grotesque et l'inacceptable. Comme Constance Rourke nous en a fait prendre consciences, les premiers spectacles de ménestrels - avec leur chorégraphie dérivée de la culture noire, le son du banjo et des os qui s'entrechoquent, des voix qui gloussent, des histoires drôles dans un pseudo-dialecte noir, des chansons absurdes, des costumes de paillettes et des acteurs en sueur - constituaient un rituel d'exorcisme. D'autres cultures blanches avaient elles aussi leurs poupées de chiffons noires [Gollywog] et leurs

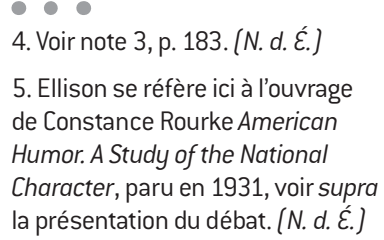
de Constance Rourke American Humor. A Study of the National Character, paru en 1931, voir supra la présentation du débat. (N. d. É.) 


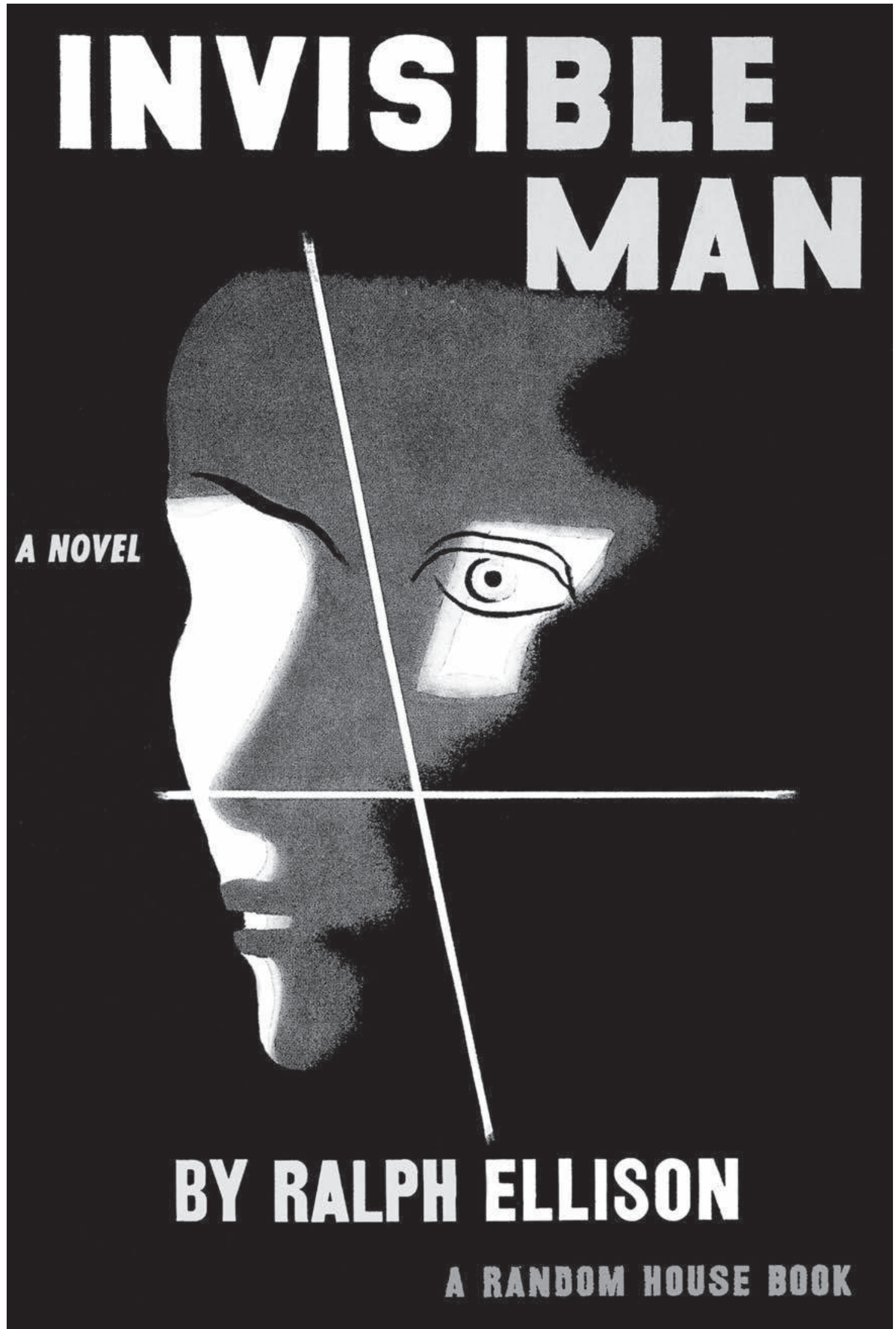

Fig. 10 Couverture originale de Invisible Man (1952), de Ralph Ellison. Dessin de Edward McKnight Kauffer. Droits réservés. 
"Maures». Mais, chez nous, le phénomène de l'esclavage est venu se loger en plein cœur du drame social américain. Aux États-Unis, la figure du Noir était par trop réelle pour qu'on lui réponde avec légèreté, bien trop sérieuse pour être traitée autrement que par un art d'ampleur nationale. Le masque était un élément inséparable de l'imagerie du pays. Dès lors, même quand un Noir jouait un rôle abstrait, le code en usage ne changeait pas. Son costume avait beau recourir au symbolisme «sacré " du drapeau américain - un pantalon et un pardessus aux bandes rouges et blanches, et un faux col bleu constellé d'étoiles -, il ne pouvait se présenter devant le public autrement qu'avec des gants blancs et un visage noirci au charbon ou à la graisse.

Ce masque, cette stylisation et cette modification délibérée du visage et des mains étaient impératifs pour que s'installe l'atmosphère dans laquelle pourrait être goûtée cette fascination pour la noirceur et pour que la catharsis soit menée à bien. L'identité raciale de l'acteur ne comptait pas. C'était le masque qui importait, à plus d'un titre. Sa fonction était de voiler l'humanité des Noirs, ainsi réduits à de simples signes, et de réprimer l'identification morale du public blanc avec ses propres actes et avec les ambiguïtés humaines présentes derrière ce masque.

Hyman souligne l'aspect comique de l'interprétation contemporaine de ce personnage : aujourd'hui, des hommes instruits et brillants parodient un grotesque infrahumain. Fort bien. Mais, lorsque nous affinons la focale en délaissant les vastes horizons des archétypes pour procéder à une inspection plus serrée, nous nous rendons compte que, concrètement, l'auto-humiliation de la figure "sacrificielle» reste toujours de mise. L'un des puissants ressorts à l'œuvre chez le public est toujours la distanciation psychologique vis-à-vis de cet acte d'automutilation. Les questions de race, de classe et de culpabilité sont ici étroitement imbriquées. L'aspect comique n'est pas séparable de l'identité raciale de l'acteur - comme cela apparaît clairement dans l'exemple que Hyman tire de Black Boy de Wright ${ }^{6}$. En assumant contre rétribution un rôle dévalorisant pour le groupe, l'acteur nourrit d'une part la croyance des spectateurs en la "noirceur " des choses noires, mais il les libère d'autre part efficacement de leur culpabilité, en participant à cette recherche du profit qui est précisément au cœur du processus ayant fait du Noir le bouc émissaire national. Il existe toutes sortes de comédies : celle du chef tribal dans Les Vertes Collines d'Afrique me vient ici à l'esprit. Malgré la honte, celui-ci ne parvient pas à dissimuler son hilarité à la vue d'une hyène touchée par balle qui sort elle-même ses intestins pour les dévorer, comme le dit Hemingway, «avec délectation».

Tout en bas, dans les sombres profondeurs du melting-pot, où le privé devient public et le public privé, où le noir est blanc et le blanc est noir, où l'immoral devient moral et où ce qui est moral n'est rien d'autre que ce qui est profitable (ou supportable), la délectation de l'homme blanc pourrait bien n'être que l'amère et secrète blessure de l'homme noir.

Il n'y a rien d'étonnant à ce que ce guignol au visage noirci qui plaît tant aux Blancs soit pour les Noirs le symbole de tout ce qu'ils rejettent dans la conception blanche de la race. Lorsqu'il apparaît par exemple sous les traits du nègre Jim, le Noir commence à étouffer. Écrivant à une époque où le ménestrel blackface était encore très populaire, peu après une guerre dont même les abolitionnistes sortirent lassés des problèmes touchant aux Noirs, Mark Twain calqua le personnage de $\mathrm{Jim}^{7}$ sur les contours de la tradition du ménestrel. Et c'est au regard de ce masque stéréotypé que nous apparaissent la dignité et l'humanité de Jim - et, par voie de conséquence, toute la complexité de Twain. Pourtant, l'ambivalence

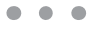

6. Roman autobiographique de l'auteur noir américain Richard Wright, publié en 1945, l'un des premiers romans écrits par des Noirs sur leurs conditions de vie. (N. d. É.)

7. Dans Adventures of Huckleberry Finn (1884). (N. d. É.) 
entre son identité de parent et d'adulte et sa naïveté "puérile " provient précisément du modèle de ménestrel à l'origine du personnage. En retour, cette tension fait paradoxalement apparaître Huck, avec sa sophistication toute citadine, plus adulte. Voilà qui trouble certainement un lecteur noir, et nous procure en outre une explication, plus terre-à-terre et moins psychanalytique, du malaise qui sourd de l'argument de Leslie Fiedler sur les relations entre Jim et Huck dans son essai Come Back to the Raft Ag'in, Huck Honey ${ }^{8}$ !

Examinons un roman plus récent pour faire valoir mon argument. Dans L'Intrus ${ }^{9}$, Faulkner met également en scène une rencontre entre un adulte noir et un gamin blanc, Lucas Beauchamp et Chick Mallison. En dépit de toutes les distinctions de races et de castes, Lucas prend l'ascendant sur le jeune Mallison du fait de sa maturité. Il refuse de se plier au duel hiérarchique de nature comique qui lui est imposé par le jeune Blanc qu'il a sauvé. Faulkner était libre de répudier la confusion entre la virilité et la caste de l'homme noir, qui est la marque de la tradition sudiste. Mais Twain, qui est plus proche de la période de la Reconstruction et de la tradition orale, pouvait plus difficilement s'affranchir de la maxime blanche qui veut que l'homme noir soit traité comme un jeune garçon ou un "oncle " - mais jamais comme un homme. L'amitié de Jim pour Huck donne l'impression d'une relation entre deux gamins plutôt que d'une amitié entre un adulte et un garçon. Dès lors, on y trouve implicitement non seulement une transgression des relations stéréotypées entre Noirs et Blancs qu'impose la société, mais aussi une violation de nos conceptions de la masculinité à l'âge adulte.

Avec Jim, les sphères privée et publique sont mises en lumière sous le jour le plus cru. Devant nous, une figure «archétypique» s'incline devant le réalisme qui est la marque du roman en tant que genre. C'est ce réalisme propre à la forme littéraire qui explique à mon sens cette ambiguïté qui gênait tant Fiedler. Lorsqu'il a qualifié l'amitié de Jim et Huck d'homosexuelle, on l'a accusé d'être un provocateur et de rechercher le sensationnalisme. Pourtant, je le crois si profondément perturbé par la façon dont sont résolues les lourdes dichotomies symbolisées par la blancheur et la noirceur, qu'il a sauté à pieds joints dans ce marécage symbolique. Lui, dont le métier est pourtant de décoder de telles situations, en a oublié au passage les contraintes propres au roman. Dès lors, embourbé jusqu'au cou, il a hurlé le terme le plus violent qu'il pût trouver pour nommer le chaos. Toutes choses égales par ailleurs, il aurait aussi bien pu parler de «viol», d'«inceste », de "parricide » ou, pire encore, d'« union interraciale ». Il est pour le moins ironique que ce qui est pour un Noir une chute ratée dans la lutte - victorieuse - de Twain avec cette figure si ambiguë en blackface soit perçu par un critique comme une perte symbolique d'identité sexuelle. J'y vois en tout cas une vraie richesse pour la littérature.

Bien que la figure du blackface me semble typiquement occidentale et calviniste, et si propre à notre terroir, Hyman la voit comme un rejeton direct de l'archétype africain du décepteur. Sans chercher à ergoter sur ce point, je me contenterai de remarquer que, même si c'était le cas, sa faculté à épouser les besoins symboliques de l'Amérique blanche est encore plus fascinante que ses origines supposées. Cette souplesse nous apprend énormément sur le fonctionnement des valeurs américaines telles que modulées par le folklore et la littérature. Nous voici une fois de plus ramenés aux questions de l'ordre et du chaos, de la réalité et de l'illusion, de l'être et du non-être.

Selon Karl Kerenyi (cité dans Paul Radin, The Trickster, Routledge, 1956), le décepteur représente une personnification du corps 


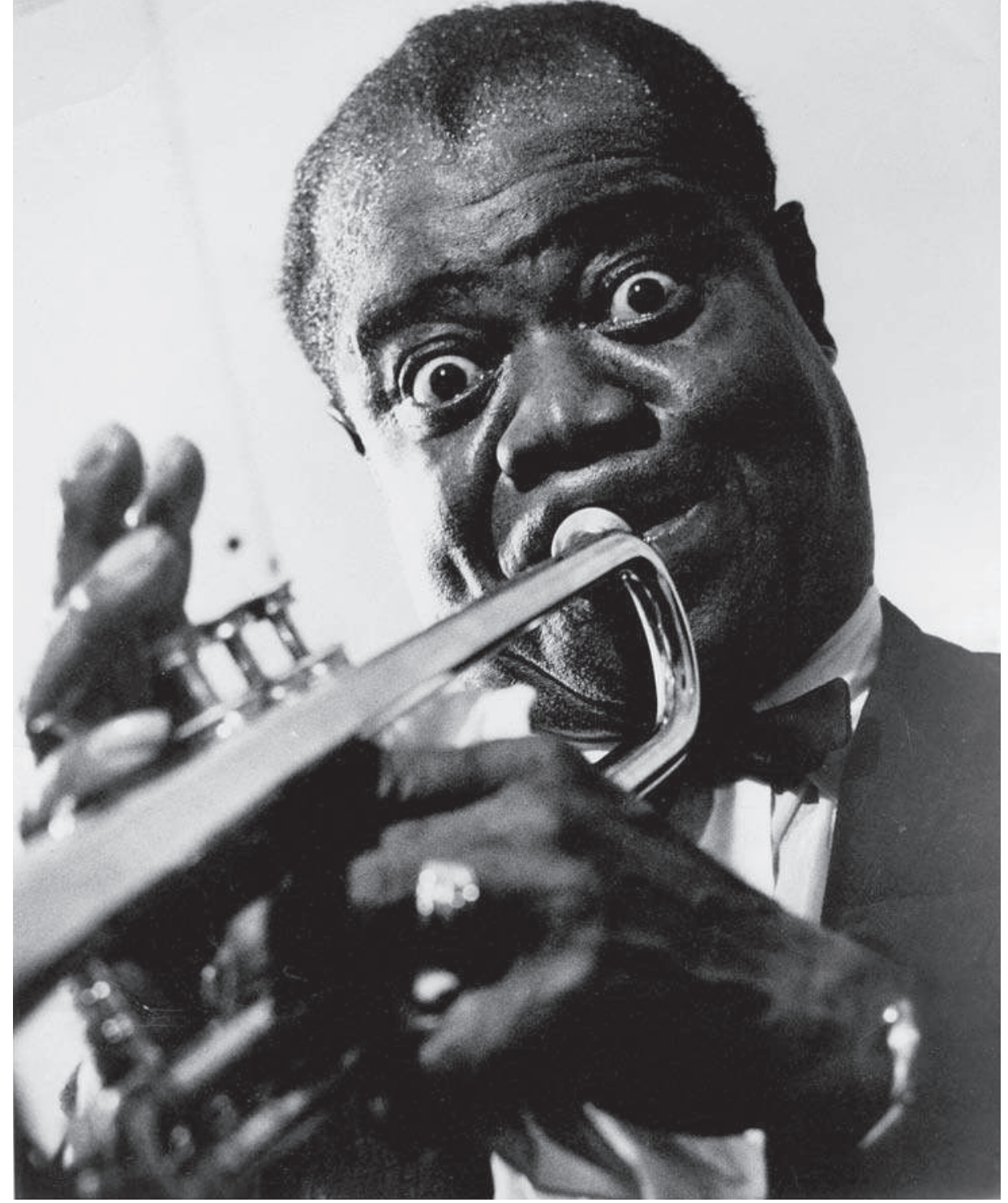

Fig. 11 Louis Armstrong, trompettiste (1901-1971). Les yeux écarquillés sont une convention classique des spectacles de ménestrel, qui traverse l'histoire de la culture populaire américaine. On la retrouve notamment chez Fats Waller et Josephine Baker. Avec la permission de Photofest.

qui n'est [...] jamais totalement soumis, qui est régi par la faim et la soif, condamné à la souffrance et aux blessures, et qui mène des actions autant rusées que stupides. Le désordre fait partie de la vie dans sa totalité [...] et le décepteur est l'esprit de ce désordre. Sa fonction au sein des sociétés archaïques, ou plutôt la fonction de son énonciation mythologique, des contes que l'on colporte sur lui, est d'ajouter du désordre à l'ordre afin d'en faire un tout, et de rendre possible une expérience du prohibé à l'intérieur des limites de ce qui est permis.

Mais notre société n'est précisément pas archaïque (bien que ses éléments archaïques exercent une influence beaucoup plus grande sur nos vies que ce qui est généralement admis). N'est-il pas ironique qu'au sein d'une société considérée comme la plus "ouverte" au monde, la marge de manœuvre de la figure du décepteur noir soit limitée par la rigidité d'attitudes racistes, par les opportunités politiques et par la culpabilité qui accompagne la frénésie des Blancs à s'identifier à l'homme de chair et d'os dont les caractéristiques raciales ont été expropriées à des fins immorales? Soit dit en passant, Hyman aurait trouvé en Louis Armstrong un bien meilleur exemple du décepteur, évoluant cette fois dans l'univers des sons plus que dans celui des mots et de la pantomime. La liberté clownesque et les pouvoirs enivrants d'un Armstrong sont des plus élisabéthains. Il prend des libertés avec rois, reines et présidents; il accentue l'épaisseur physique de sa musique avec 
force grimaces, sueurs et crachats; il réalise la prouesse magique de faire sortir des mélodies romantiques de son gosier rocailleux; et, il y a quelques années encore, il recommandait à tous son médicament personnel, «l'eau de Pluton", comme un remède puissant pour la santé, le bonheur, et la paix dans le monde.

Lorsque le Blanc se glisse derrière le masque du décepteur, il voit sa liberté limitée par la peur de passer de la dimension symbolique de cette personnification du chaos à l'ordre du réel. Il est terrifié à l'idée de rester enfermé quelque part dans les mystères de l'enfer (car il y a un mystère dans la blancheur de la noirceur, dans l'innocence du mal et dans la culpabilité de l'innocence, que les Noirs, en tant qu'initiés, savent tourner en dérision dans les blues). Il est terrifié à l'idée de perdre cette liberté dont il s'arroge le monopole, dans une société si rapide et fluide, délestée du poids de la tradition comme des limitations de classes.

Un autre aspect ironique du vieux problème américain de l'identité affleure ici. En contrefaisant l'identité noire, l'homme blanc se met à douter de l'authenticité de l'image qu'il a créée de lui-même. Après tout, il a réellement commencé à devenir américain lorsque, refusant l'impôt royal et se révoltant, il eut recours à un masque qui lui était propre. Et, lorsque nous examinons nos tourments identitaires à la lumière de ce que Robert Penn Warren a appelé la nature «intentionnelle » de nos premiers pas en tant que nation, la citation suivante de W. B. Yeats prend tout son sens :

Il y a une relation entre la discipline et le sens théâtral. Si nous ne parvenons pas à nous imaginer autrement que nous sommes et à assumer un second moi, nous ne pouvons nous imposer une discipline, bien que nous en acceptions une de la part des autres. La vraie vertu est aux antipodes de l'acceptation passive d'un code en usage. Elle est un masque que l'on porte, c'est là la condition d'une vie pleine et difficile.

Pour les colons nouvellement affranchis de la tutelle britannique, l'affirmation d'une identité américaine revenait à assumer un masque. Ainsi, la discipline d'une conscience de soi nationale s'imposait peu à peu. Mais, surtout, cela permit aux Américains de prendre conscience du hiatus ironique qui se love toujours entre l'apparence et la réalité, entre les discontinuités de la tradition sociale et ce besoin irrépressible d'une reconstruction du passé - et peut-être même le pressentiment que la société est la création de l'homme et non pas celle de Dieu. Les Américains inaugurèrent leur révolte contre la patrie anglaise quand, déguisés en Indiens, ils déversèrent des cargaisons de thé dans le port de Boston. La mobilité de la société créée dans cet espace infini a sans cesse encouragé depuis le recours au masque, pour le meilleur comme pour le pire. Comme l'industrie publicitaire le montre clairement - une industrie entièrement consacrée à la création de masques -, ce qui ne peut imposer son autorité par le recours à la tradition peut l'emprunter grâce au masque. Le masque est un jeu sur les possibles et l'Amérique est précisément une société qui en offre énormément. C'est lorsque notre vie est la plus américaine qu'elle est la plus théâtrale.

Et c'est pourquoi je ne peux accepter que Hyman qualifie le rôle du «type intelligent qui fait l'idiot" comme fondamentalement noir, s'il entend par "situations conflictuelles » ces moments où la question raciale l'emporte sur tout le reste. À la vérité, c'est un rôle que les Noirs partagent avec d'autres Américains, et c'est peutêtre le plus «Yankee» d'entre tous. C'est une stratégie basique de la culture, renforcée par notre anti-intellectualisme, notre tendance à la conformité et l'aspiration corollaire de l'individu à la tranquillité; et même, plus prosaïquement, par l'envie de faire un peu de profit. Mais, à la base, la stratégie s'enracine dans la conscience 
du canular qui est au cœur de l'identité américaine. Un vieil ami sudiste au teint très sombre m'a rapporté ce dialogue qu'il eut avec un chef d'entreprise blanc qui se plaignait de sa dureté en affaires. Il lui répondit en rigolant : «Je sais, tu pensais que j'étais Noir, pas vrai? » C'est au travers de cette blague qu'aux États-Unis Noirs et Blancs réussissent à socialiser. L'Américain blanc a attribué au Noir une absence totale de passé ou de tradition (voilà qui frappe le Blanc d'horreur), exactement de la même façon que l'Européen (et le critique américain nostalgique de la stabilité perdue des cultures de l'Ancien Monde) le considère lui-même. Mais le Noir américain sait pertinemment que le Blanc comme le Noir ont été nourris au même sein, celui de l'Amérique. Que dire de plus? Chacun est intimement persuadé que lui seul sait ce qui est valide dans l'expérience américaine, et que l'autre sait qu'il sait mais ne l'admettra pas. Tous deux suspectent l'autre d'être à l'origine d'une contrefaçon.

À moitié conscient que la représentation qu'il se fait du Noir est fausse, le Blanc est sur la défensive et suspecte le Noir de chercher à le rouler sans cesse en le supposant animé par la peur et la colère - ce qui est le cas la plupart du temps. De l'autre côté du miroir, le Noir observe le Blanc et se demande comment il est possible que les « Gris »-le terme que les Noirs utilisent pour désigner les Blancs - se bercent d'autant d'illusions quant au caractère inextricable de la noirceur et de la blancheur. Pour lui, l'homme blanc est un hypocrite qui se targue d'une identité pure tout en vantant son humanisme au monde entier.

Très souvent, pourtant, le fait que le Noir revête le masque n'est pas tant motivé par la peur que par un profond rejet de l'image créée pour usurper son identité. Parfois, c'est tout simplement pour le plaisir du jeu; parfois, c'est pour défier ceux qui se targuent, par-delà la distance psychologique imposée par les mœurs raciales, de connaître son identité. Mais voilà qui est en tout cas typiquement dans la veine américaine. Benjamin Franklin, le scientifique pragmatique, l'homme d'État chevronné et l'amateur raffiné, laissa les Français s'imaginer qu'il était sorti tout droit de l'état de nature. Hemingway pose en sportif illettré, Faulkner en fermier; Abe Lincoln laissa les gens le prendre pour un simple avocat provincial... jusqu'au moment fatidique. Ici, le numéro du "noiraud » ["darky" $a c t$ nous rend tous frères. L'Amérique est un pays de joueurs qui portent le masque. Nous le portons à des fins d'agression comme de défense, lorsque nous nous projetons dans le futur et que nous voulons sauver le passé. En bref, les motifs cachés derrière le masque sont aussi nombreux que les ambiguïtés que le masque dissimule.

Le fond de ma controverse avec Hyman ne porte pas tant sur ses idées quant à l'importance de la tradition vernaculaire, ni sur son intérêt pour les archétypes, mais plutôt sur sa tendance à détourner, lorsqu'il parle de littérature, le contenu des œuvres pour qu'elles se plient à sa théorie. Il se réfère par ailleurs à mon roman, prenons-le donc au mot. Hyman est tellement obsédé par les formes dérivant d'archétypes qu'il ne parvient pas à voir que le grand-père du narrateur dans Homme invisible n'est pas plus engagé dans un numéro de «noiraud " que ne l'était Ulysse dans la grotte de Polyphème. Il n'est d'ailleurs pas non plus ce « type intelligent qui fait l'idiot ", mais un homme faible qui connaît les faiblesses de ses oppresseurs. Il y a une profonde amertume chez ce vieil homme, la même qui gagnera bientôt son petit-fils, et la stratégie qu'il lui conseille est une sorte de jiujitsu de l'esprit, un rejet et un refus qui prend les habits du « Yes Sir! ». Samson, les yeux crevés à Gaza, fit s'écrouler l'édifice lorsque ses forces lui revinrent; faible politiquement, le grand-père a appris que le conformisme menait à une fin similaire, et il en fait part à ses enfants. Dès lors, son masque d'humilité contient la 
sagesse de celui qui a appris le secret de dire des «oui " qui ont l'effet des "non " les plus éloquents. Ici aussi, nous trouvons le rejet d'un code en usage et ce refus prend une connotation métaphysique. Plus important encore dans l'économie du roman, il représente l'ambiguité du passé pour le héros, pour qui son conseil murmuré sur le lit de mort sonne comme l'énigme du Sphinx et oriente l'intrigue dans une double direction qu'il tentera de suivre tout au long du roman.

B. P. Rinehart (P. pour "Protée » [Proteus] et B. pour «Béatitude» [Bliss]) tombe certainement à point nommé pour Hyman et sa quête de la figure du décepteur. Voilà un homme rusé qui force l'admiration de ceux qui estiment le maquignonnage et le savoir-faire, un virtuose américain de l'identité qui glisse sur le chaos et les changements rapides; il est cupide, car sa mascarade est motivée par l'argent comme par le pur plaisir de l'usurpation d'identité; il est divin, car il apporte de nouvelles techniques comme les guitares électriques au service de Dieu, et parce qu'il fait jouer de nombreuses images dans son personnage tout en restant lui-même invisible; il est phallique dans son rôle d' "amant "; quand il fournit des numéros gagnants pour des paris clandestins, il représente une manne et fait des miracles, car il transforme (pour les gagnants, bien sûr) des centimes en dollars et nourrit (et se nourrit) des pauvres. On pourrait ainsi facilement allonger la liste à la manière de cette critique racoleuse pour qui la mythologie est un fond de commerce, jusqu'à ce que la fiction se dissolve dans l'anthropologie. Mais le rôle de Rinehart dans la structure formelle du roman est de suggérer au héros une échappatoire dans son combat avec Ras, et un moyen d'appliquer le conseil énigmatique de son grand-père à sa propre situation. Si l'on projetait Rinehart dans le panthéon de la littérature, il y côtoierait d'illustres aînés comme Felix Krull chez Mann, le baron Clappique de Malraux dans La Condition humaine et bien d'autres encore, mais ce serait là s'adonner à un exercice critique un peu vain, et cela n'apporterait finalement pas grand-chose au débat.

L'identité des personnages de fiction est déterminée par le réalisme implicite de la forme. Ils ne l'héritent pas de la tradition; ils sont ce qu'ils font ou ne font pas. Les archétypes sont hors du temps, les romans hantés par le temps. Les romans parviennent parfois à s'affranchir d'une époque dans la longue durée, mais, si les symboles qui habitent l'œuvre rejoignent ces mythes universels, ils ne le font que depuis la texture particulière d'une forme singulière de réalité sociale. Dans l'épilogue, l'homme invisible ne se dissimule pas dans une obscurité aux connotations anglo-saxonnes, mais fait entendre une voix énonçant une humble sagesse issue de sa propre introspection, après qu'il a accompli sa conversion d'agitateur en écrivain. Si, par ailleurs, le héros se prête ici à un "numéro de noiraud ", il n'est certainement pas ce type intelligent qui fait l'idiot. Car le roman - ses mémoires - n'est qu'une longue diatribe hurlée à la face du monde, puis étouffée dans un éclat de rire. Ici, il ne dissimule pas, il confesse. Sa mobilité est double, géographique comme le remarque Hyman, mais, plus fondamentalement, intellectuelle. Et, fidèle en cela au renversement anglais de l'intrigue, tout autant qu'à la conception noire américaine de la noirceur, son mouvement vertical et descendant (pas dans un "égout ", n'en déplaise à Freud, mais dans une cave à charbon, source de chaleur, de lumière, de pouvoir et, en vertu des motivations du personnage, d'autoperception) est un processus d'ascension vers une compréhension de sa condition humaine. Ce ne sont pas les blues ni la sociologie qui expliquent sa mobilité insatiable, mais une forme littéraire qui a le temps et le changement social pour province. L'insatiabilité est en outre une qualité des plus américaines, qui transcende la géographie, la sociologie et l'ancien état de servitude. 
Les discussions sur la littérature et la tradition folklorique qui font l'impasse sur la spécificité des formes littéraires mobilisées me semblent donc sujettes à caution. La plupart des écrivains que mentionne Hyman sont des romanciers, des artisans aux prises avec une forme qui a absorbé la tradition vernaculaire dans ses structures thématiques, ses intrigues, son symbolisme et sa rhétorique, et qui a développé ses propres stratégies avec le folklore comme avec les mœurs, l'histoire, la sociologie et la psychologie. En outre, les romanciers actuels sont plus enclins à être influencés par la lecture d'autres romans que par leurs affinités avec un quelconque folklore.

J'ai recours au folklore dans mon travail non pas parce que je suis Noir, mais parce que des écrivains comme Eliot et Joyce m'ont fait prendre conscience de la valeur littéraire de mon héritage folklorique. Mon environnement culturel, comme la plupart des Américains, est double (mon second prénom, et laissezmoi vous assurer que cela n'a rien de léger, est Waldo ${ }^{10}$ ).

Des décepteurs comme Ulysse m'ont accompagné depuis aussi longtemps que le lapin futé de la tradition noire américaine, et il m'était plus facile de m'imaginer en petit Ulysse qu'en lapin, nonobstant ma race, mon humanité ou même le caractère débordant de mon imagination. Plus tard, je m'identifiais avec Huck Finn (c'est le surnom que je donnais à mon frère), mais pas, et ce malgré les apparences, avec le Nègre Jim, qui m’apparaissait plutôt comme le portrait inadéquat qu'un Blanc pouvait se faire d'un esclave.

Ce que je veux dire ici est que l'écrivain noir américain est également l'héritier de cette expérience humaine qu'est la littérature, et cela pourrait s'avérer bien plus important pour lui qu'une quelconque tradition folklorique, même vivante. Pour moi tout au moins, dans une culture américaine discontinue, diverse et si changeante, la stabilité de la tradition vernaculaire noire américaine ne s'avéra précieuse que lorsqu'elle fut l'occasion d'une découverte littéraire. Prise comme un tout - avec ses spirituals, ses blues, son jazz et ses contes ancestraux-, cette tradition a, comme le suggère Hyman, beaucoup à nous apprendre sur la confiance, l'humour et le pragmatisme nécessaires pour vivre dans un monde qui, dans son insécurité et son absurdité blues, ressemble beaucoup à celui des créateurs de la tradition folklorique. Pour ceux qui sont capables d'exprimer ses significations dans un vocabulaire plus ample et plus précis, elle a en effet beaucoup à offrir. Hyman nous rend donc un service lorsqu'il nous fait prendre conscience que la tradition folklorique noire américaine représente une source pour la littérature qu'il convient de ne pas négliger. Mais pour le romancier, de quelque culture ou de quelque phénotype soit-il, la forme spécifique de son art est pour lui sa plus grande liberté, et ses idées sur le monde lui appartiennent en propre.
-

10. Le père d'Ellison - un ouvrier aventurier et entrepreneur épris de lecture qui décéda d'un accident du travail lorsque Ellison avait trois ans - a choisi les prénoms de son premier fils en hommage au poète philosophe et libéral américain Ralph Waldo Emerson. Ellison rappelle dans un essai au titre évocateur, Hidden Name and Complex Fate, que cela faisait rire les Noirs de son entourage lorsqu'il passait les voir comme delivery boy, et qu'il mit lui-même un certain temps à assumer cet héritage «blanc ». (N. d. T.) 


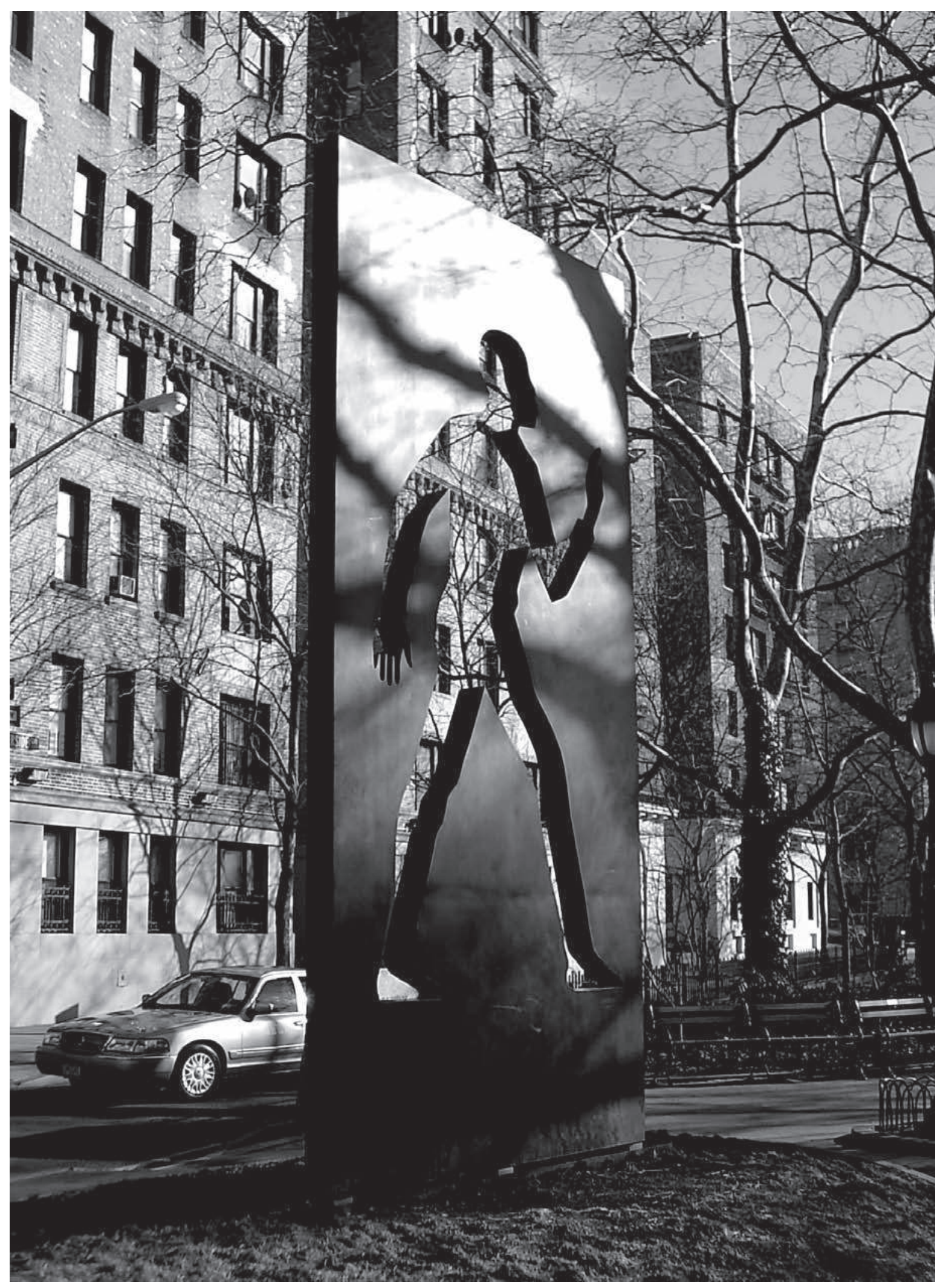

Fig. 12 Mémorial Ralph Ellison, au 730 Riverside Drive, New York City, en bas de son lieu de résidence de 1954 à sa mort en 1994. Photo Emmanuel Parent. 